Research Article

\title{
Characteristics and Functions of the Rumen Microbial Community of Cattle-Yak at Different Ages
}

\author{
Yuzhu Sha $\mathbb{D}^{1},{ }^{1}$ Jiang Hu, ${ }^{1}$ Bingang Shi, ${ }^{1}$ Renqing Dingkao, ${ }^{2}$ Jiqing Wang, ${ }^{1}$ Shaobin Li, \\ Wei Zhang, ${ }^{1}$ Yuzhu Luo, ${ }^{1}$ and Xiu Liu ${ }^{1}{ }^{1}$ \\ ${ }^{1}$ College of Animal Science and Technology/Gansu Key Laboratory of Herbivorous Animal Biotechnology, \\ Gansu Agricultural University, Lanzhou 730070, China \\ ${ }^{2}$ Gannan Institute of Animal Husbandry Science, Hezuo 747000, China
}

Correspondence should be addressed to Xiu Liu; liuxiu@gsau.edu.cn

Received 25 November 2019; Revised 30 January 2020; Accepted 19 February 2020; Published 4 March 2020

Academic Editor: Kok Tat Tan

Copyright (c) 2020 Yuzhu Sha et al. This is an open access article distributed under the Creative Commons Attribution License, which permits unrestricted use, distribution, and reproduction in any medium, provided the original work is properly cited.

\begin{abstract}
A cattle-yak, which is a hybrid between a yak (Bos grunniens) and cattle (Bos taurus), is an important livestock animal, but basic questions regarding its physiology and environmental adaptation remain unanswered. To address this issue, the present study examined the species composition and functional characteristics of rumen microorganisms in the cattle-yak of different ages ( 2 and 3 years old) by metagenomic analysis. We found that rumen microbial community composition was similar at the two ages. Firmicutes, Fibrobacteres, Euryarchaeota, Bacteroidetes, and Proteobacteria were the predominant phyla, with Firmicutes accounting for the highest percentage of bacteria in 2-year-old (48\%) and 3-year-old (46\%) animals. Bacterial species involved in lignocellulose degradation were detected in the rumen of adult cattle-yaks including Ruminococcus flavefaciens, Ruminococcus albus, Fibrobacter succinogenes, and Prevotella ruminicola, with $F$. succinogenes being the most abundant. A total of 145,489 genes were annotated according to the Carbohydrate-active Enzyme database, which identified glycoside hydrolases as the most highly represented enzyme family. Further functional annotation revealed specific microflora and genes in the adult rumen that are potentially related to plateau adaptability. These results could explain the heterosis of the cattle-yak and provide insight into mechanisms of physiologic adaptation in plateau animals.
\end{abstract}

\section{Introduction}

Ruminants have a diverse microbiota in their rumen including bacteria, fungi, archaea, protozoa, and viruses. These microorganisms can degrade the plant cell wall and fibrous substances that are converted into absorbable compounds such as proteins and volatile fatty acids (VFAs) $[1,2]$. One study analyzing the rumen microbiome of Indian buffalo identified 2614 contigs encoding putative degradative enzymes [3], and another reported 42 operational taxonomic units representing the rumen bacterial community of adult buffalo [4]. Microbiome profile can vary according to the developmental stage; for instance, rumen microbial community composition in cows changes markedly from birth to the age of 2 years [5]. The rumen microbial environment of adult cattle is more stable and stricter than that of newborn calves, although it is unclear whether it also changes throughout adulthood.

A cattle-yak is the F1 hybrid between the female yak (Bos grunniens) and male cattle (Bos taurus), with the male cattleyak being sterile. Cattle-yaks exhibit greater adaptability to harsh environments and provide more meat per weight than yak [6], along with milk, wool, fuel, and other products for communities living in the Qinghai-Tibet Plateau, making it an important livestock animal. Cattle-yaks live at high altitudes and feed mostly by grazing; they can also grow normally during the dry grass period of the Plateau when the food supply is limited. The rumen of cattle-yaks is presumed to contain microorganisms with lignocellulose-degrading capability that enable adaptation to the plateau environment [7], but few studies have investigated rumen microbiota community composition and function in these animals. 
TABLE 1: Sequence data for analyzed samples.

\begin{tabular}{lcccccc}
\hline Sample & Raw reads & Clean reads & Cleanper (\%) & Clean GC (\%) & filtHost reads & filtHost GC (\%) \\
\hline 2-year -old & $102,395,772$ & $101,320,532$ & 98.51 & 42.78 & $84,611,980$ & 44.03 \\
3-year-old & $83,452,932$ & $83,117,032$ & 99.59 & 48.32 & $81,812,068$ & 48.53 \\
\hline
\end{tabular}

Raw reads: No. of raw reads; clean reads: No. of clean reads; Cleanper (\%): proportion of clean reads; clean GC (\%): GC content of clean reads; filtHost reads: No. of filtered host reads; filtHost GC (\%): GC content of filtered host reads.

To address this issue, we carried out a metagenomic analysis of the rumen microbiome of cattle-yak at two different ages ( 2 and 3 years of age). Our results reveal the diversity of the rumen microbial community in ruminants and suggest potential mechanisms of adaptation to the unique plateau environment.

\section{Materials and Methods}

2.1. Ethics Statement. Experiments involving animals were carried out in accordance with regulations for the Administration of Affairs Concerning Experimental Animals (Ministry of Science and Technology, China; revised in June 2004). Sample collection was carried out according to the guidelines of the Ethics Committee for the Care and Use of Laboratory Animals of Gansu Agricultural University.

2.2. Animals and Sample Collection. Six male 2- and 3-yearold healthy cattle-yaks (yak $+\times$ Jersey cattle ${ }^{-}, n=3$ each) were obtained from a farm in Gannan Tibetan Autonomous Prefecture (Gansu Province, China). Rumen samples $(50 \mathrm{ml}$, containing fluid and feed particles) were collected from each animal after slaughter and immediately frozen in liquid nitrogen and stored at $-80^{\circ} \mathrm{C}$ until use.

2.3. DNA Extraction and High-Throughput Sequencing. Genomic DNA was extracted from rumen fluid and purified using the TIANamp Stool DNA Kit (Tiangen Biotech, Beijing, China) according to the manufacturer's instructions and sent to Gansu Meita Biomedical Co. (Lanzhou, China) for high-throughput sequencing.

\subsection{Bioinformatics and Statistical Analyses. Genomic DNA} was sheared with a Covaris M220 focused ultrasonicator (Thermo Fisher Scientific, Waltham, MA, USA), yielding $\sim 300 \mathrm{bp}$ fragments for library construction. Highthroughput sequencing was performed on the HiSeq 2000 platform (Illumina, San Diego, CA, USA). Low-quality and unidentified $(\mathrm{N})$ bases were filtered using fastp software; clean reads were combined with the host reference genome sequence, and host contaminating sequences were removed by Burrows-Wheeler alignment (bwa) [8]. Megahit software was used for metagenomic contig assembly from single and mixed samples. Open reading frames (ORFs) and amino acid sequences were predicted with MetaGeneMark software, and a nonredundant gene set was obtained using CD-HIT-est [9]. We used information for each sample read and assembly results to compare nonredundant gene sets, and gene abundance was analyzed using bwa+featureCounts software.
TABLE 2: Assembly and nonredundant gene prediction results.

\begin{tabular}{lc}
\hline Item & Number \\
\hline Number of scaffolds & $1,365,143$ \\
Total length (bp) & $1,396,336,337$ \\
N50 scaffold (bp) & 993 \\
N90 scaffold (bp) & 556 \\
Maximum scaffold (bp) & 410,268 \\
Mean length (bp) & 1022.85 \\
Number of genes & $1,281,665$ \\
Total length & $815,962,296$ \\
N50 length & 708 \\
Maximum length & 26,820 \\
Mean length & 636.64 \\
\hline
\end{tabular}

2.5. Functional Annotation. Functional annotation based on Kyoto Encyclopedia of Genes and Genomes (KEGG), Evolutionary Genealogy of Genes: Nonsupervised Orthologous Groups (eggNOG), and Carbohydrate-active Enzyme (CAZy) databases was performed using Diamond, eggNOG-mapper, and KOBAS software packages.

\section{Results}

3.1. DNA Sequence Data and Microbial Diversity Analysis. A total of $102,395,772$ and $83,452,932$ unique reads were obtained from rumen samples of 2- and 3-year old cattleyaks, respectively (Table 1). Over $98 \%$ of the original data were selected as clean reads. The GC content was higher for 3-year-old than for 2-year-old cattle-yak. The clean reads were filtered based on the host genome, yielding filtered host reads that constituted a total of $0.8 \mathrm{~GB}$ for both ages, with GC content being higher in the older animals. Contigs for single and mixed samples were combined and interrupted from $\mathrm{N}$ junctions to obtain sequences containing no $\mathrm{N}$, and fragments $<500 \mathrm{bp}$ were removed by filtering to obtain a final assembly of $1,365,143$ scaffolds constituting $\sim 1369 \mathrm{Mbp}$ (Table 2). Based on the mixed-assembly ORF prediction results, CD-HIT software was used to obtain a nonredundant gene catalog. A total of 1,281,665 nonredundant genes and $\sim 815 \mathrm{Mbp}$ nonredundant gene sequences were predicted, which had an average length of $636.64 \mathrm{bp}$.

3.2. Analysis of Rumen Microbial Community Composition. Microbial abundance in the rumen of 2- and 3-year-old cattle-yaks was analyzed with metaphin2 software according to taxonomic classification (Figure 1). At the kingdom level, bacteria and archaea were the most highly represented taxa, with the former being predominant at 2 and 3 years of age 


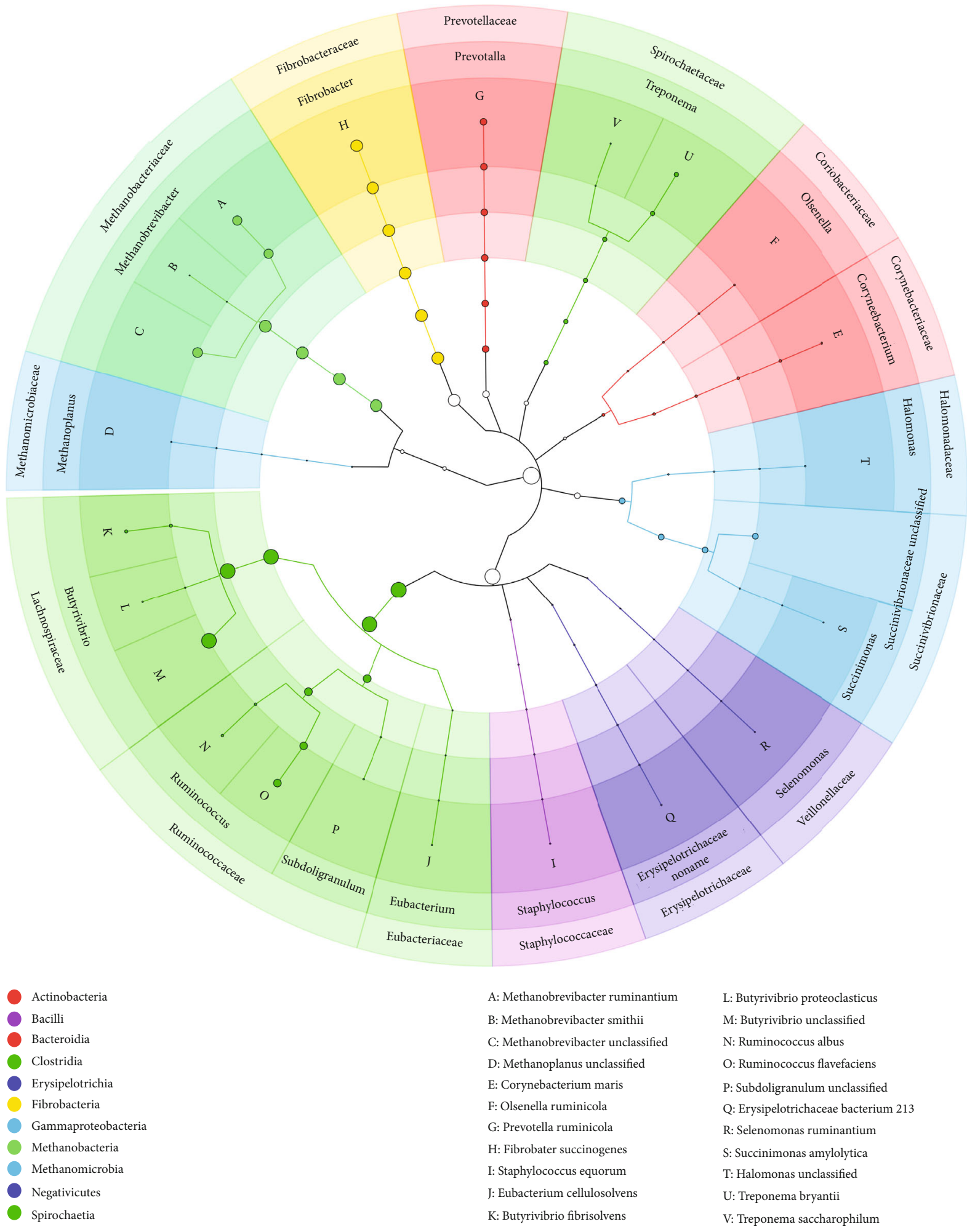

Figure 1: Cladogram of bacterial species in the rumen of cattle-yak.

(bacteria: $85 \%$ and $90 \%$ and archaea: $15 \%$ and $10 \%$, respectively) (Additional file (available here)).

At the phylum level, the rank order of abundance was Firmicutes $>$ Fibrobacteres $>$ Euryarchaeota $>$ Bacteroidete$\mathrm{s}>$ Proteobacteria at both ages. The abundance of Firmicutes was $48 \%$ at 2 years and $46 \%$ at 3 years of age (Figure 2). With the exception of Proteobacteria, there were no differences in the abundance of the other phyla between the two groups. In 2-year-old cattle-yaks, Proteobacteria represented $1 \%$ of total bacteria compared to $4 \%$ in 3-year-old samples $(P<0.001)$. At the species level, Butyrivibrio (unclassified) was the most highly represented taxon at both ages (8\%), 

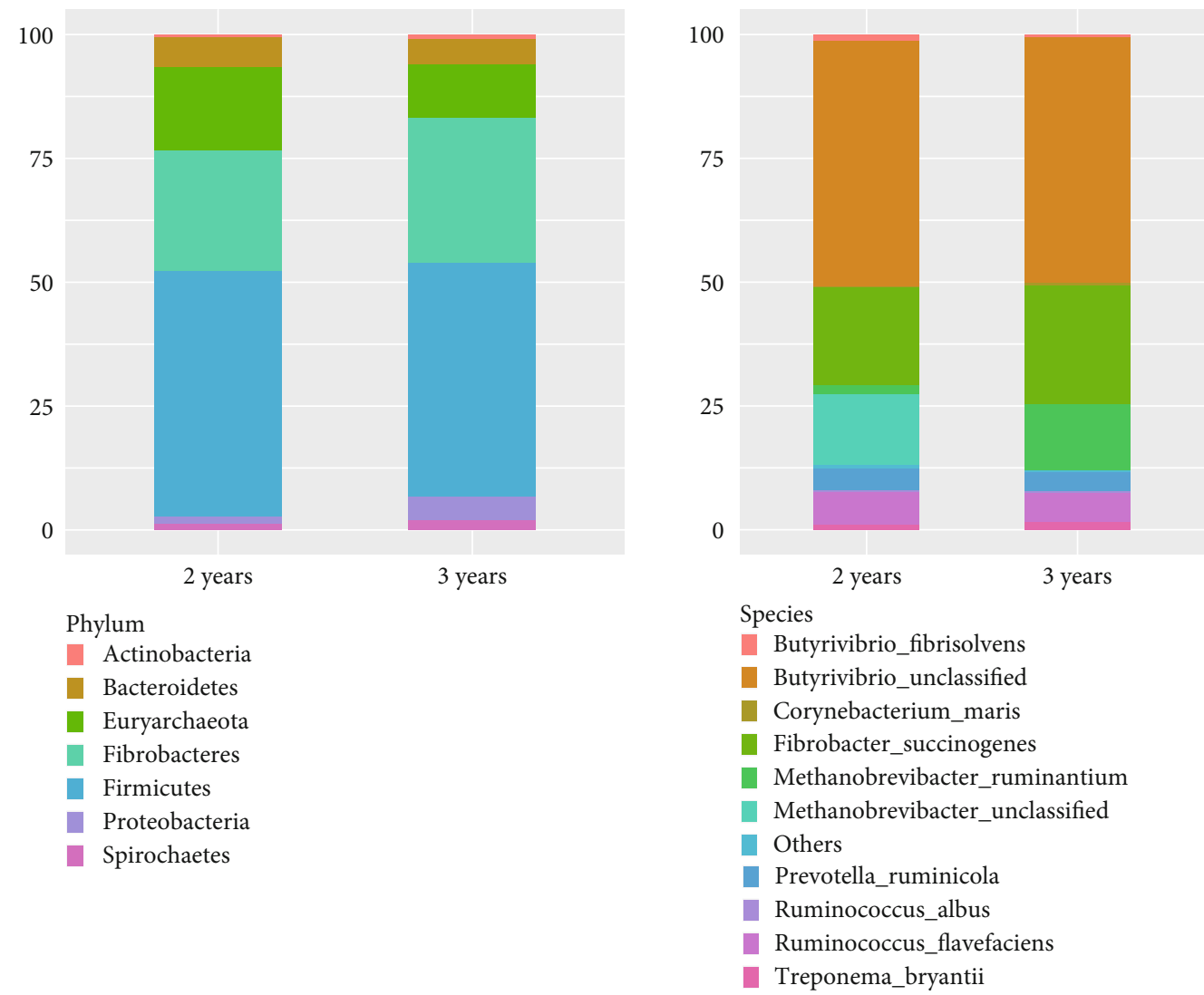

Figure 2: Rumen microbiota community composition at the phylum and species levels in 2- and 3-year-old cattle-yaks.

TABLE 3: Functional annotation results.

\begin{tabular}{lc}
\hline Total number of genes & $1,281,665$ \\
\hline Annotated to KEGG ortholog/number of orthologs & $234,104(18.27 \%) / 3196$ \\
Annotated to KEGG pathway/number of pathways & $234,104(18.27 \%) / 369$ \\
Number of genes annotated to eggNOG/number of orthologs & $805,533(62.85 \%) / 22,766$ \\
Annotated to CAZy family & $145,489(11.35 \%)$ \\
Annotated to ARDB gene/number of ARDB types & $275(0.02 \%) / 16$ \\
Number of genes annotated to VFDB/number of VFs & $99,767(7.78 \%) / 809$ \\
\hline
\end{tabular}

ARDB: Antibiotic Resistance Genes Database; CAZy: Carbohydrate-active Enzyme; eggNOG: Evolutionary Genealogy of Genes: Nonsupervised Orthologous Groups; KEGG: Kyoto Encyclopedia of Genes and Genomes; VF: virulence factor; VFDB: virulence factor database.

followed by Fibrobacter succinogenes. Additionally, multiple species related to lignocellulose degradation were detected including Ruminococcus flavefaciens, Ruminococcus albus, F. succinogenes, and Prevotella ruminicola, which were present in similar numbers in the two groups. In contrast, Methanobrevibacter ruminantium was more abundant in 3year-old $(2 \%)$ as compared to 2-year-old $(0.3 \%)$ cattle-yaks, whereas Methanobrevibacter (unclassified) was not detected in the older animals.

3.3. Annotation of Gene Function. Functional annotation was performed for 1,281,665 nonredundant genes based on the KEGG, eggNOG, and CAZy functional databases; $62.85 \%$ of the genes were annotated to eggNOG, and $11.35 \%$ were annotated to the CAZy database (Table 3).
3.3.1. CAZy Functional Annotation. A total of 145,489 genes were aligned to the CAZy database. Glycoside hydrolase (GH) genes were the most highly represented $(26,207)$, followed by glycosyltransferases (GTs; 13,218), carbohydrate esterases (CEs; 8615), and carbohydrate-binding modules (CBMs; 6452) (Figure 3). On the other hand, there were few genes associated with auxiliary activities (AA) and encoding polysaccharide lyases (PL) (1746 and 1863, respectively). The GH genes were distributed in various gene families, with most (>1500) belonging to the GH2, GH43, GH13, and GH5 families and GH47 and GH96 families accounting for only one gene each (Additional file). There were over 2000 genes belonging to the GT2, GT4, GT27, GT5, and GT41 families. CE1, CE7, CE10, CBM6, CBM32, and CBM50 families were the most highly represented among 


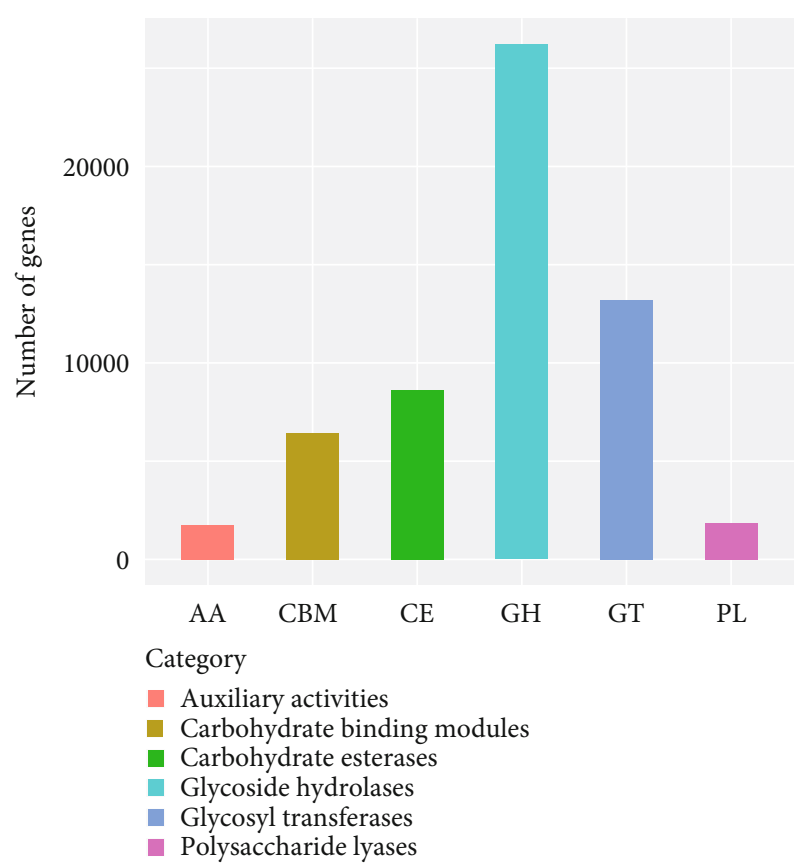

Figure 3: CAZy functional annotation of identified genes.

CE and CBM genes. In addition, 29 cohesin and 415 docker genes were identified in the rumen of cattle-yaks. The functions associated with CAZymes were similar in the two age groups, but there were minor differences: except for GTs, the other five types of CAZymes were more highly expressed in the older animals (Additional file, Figure S1).

3.3.2. eggNOG Functional Annotation. Most of the genes identified in the rumen of cattle-yaks $(54,817)$ were ascribed to the carbohydrate transport and metabolism category in the eggNOG database (Figure 4). In the two major categories of cellular processes and signaling and information storage and processing, the most abundant functions of the enriched genes were cell wall/membrane/envelope biogenesis (51,490 genes) and replication, recombination, and repair (53,011 genes). There were no differences between the two age groups in terms of the proportion of genes in these categories, although the number of carbohydrate transport and metabolism-related genes increased with age $(P<0.01)$ (Additional file, Figure S2). The proportion of genes classified under energy production and conversion, amino acid transport and metabolism, and inorganic transport and metabolism also increased with age, but the differences between the two groups were nonsignificant.

3.3.3. KEGG Functional Annotation. A total of 234,104 genes were enriched into 369 KEGG pathways (Additional file, Figure S4). The top $10 \mathrm{KEGG}$ pathways represented by these genes were related to the metabolism of small molecules-i.e., biosynthesis of amino acids, purine metabolism, carbon metabolism, and pyrimidine metabolism (Table 4). There were no differences in the proportion of genes in each pathway between age groups except for the decreased abundance of genetic information processing-related pathways and increase in metabolism pathways (Additional file, Figure S3).

\section{Discussion}

Our metagenomic analysis of rumen microorganisms of cattle-yaks of different ages revealed that bacteria were the most highly represented group (85\%), with a much lower abundance of archaea. This is consistent with the previous finding that more than half of rumen microorganisms are bacteria, which play an important role in host nutrition, physiology, and immunity [10]. Notably, the archaea content in the rumen of cattle-yaks observed here was higher than that reported in an earlier study of yaks $(2.27 \%)$ [11].

Rumen microbial community composition varies with the developmental stage [5]. In this study, the rumen microbe profiles of 2- and 3-year-old cattle-yaks were essentially the same at the phylum level. Firmicutes were the most highly represented phylum, followed by Fibrobacteres and Euryarchaeota. It was previously shown that these taxa are more abundant in cattle-yaks than in grazing yaks, possibly reflecting differences in adaptation to the unique plateau environment [11]. Under natural grazing conditions, yaks harbor a large number of cellulolytic bacteria such as Fibrobacter, which is the major degrader of cellulose plant biomass in the intestine of herbivores $[11,12]$. The higher content of Fibrobacter in the rumen of cattle-yaks compared to yaks in the same environment indicates that the former have a greater capacity to digest fibrous material, which translates into higher production efficiency. The yak emits relatively low levels of methane, a feature that is closely related to the presence of Methanobacteria (phylum Euryarchaeota), and the Methanobacter content of cattle-yaks in this study $(61 \%-63 \%)$ was lower than that of yaks $(82.13 \%)$, indicating that the hybrid animal is superior with respect to methane emission [11].

In addition, Jami et al. also found that from the first 3 days of life to adulthood at age 2, the content of Bacteroides increased significantly with age; however, we found that although the abundance increased after 2 months of age, it remained at a certain level. This study found that at 2 and 3 years old, Bacteroidetes content was $4 \%$. The major phyla represented in the intestine of mammals including humans and felines are Firmicutes and Bacteroides [13-16]; in the rumen of Holstein cow and buffalo, the major phylum is Bacteroides [17, 18]. In the rumen cattle-yak, Firmicutes and Fibrobacteres were the main bacteria; the relatively low Bacteroides content may be attributable to diet, species, season, and geographic location $[19,20]$. Plateau yaks have a higher proportion of Firmicutes (45\%) than Bacteroides (39.6\%) in the intestine [21], and a similar trend has been reported in the plateau pika [22]. It was shown in ruminants that the proportion of Clostridium (phylum Firmicutes) increased with crude fiber content in hay [23]. In addition, the Firmicutesto-Bacteroidetes $(F / B)$ ratio was found to be higher in the rumen of cows that were fed hay as compared to grain [24]. The $F / B$ content of cattle-yaks in this study was higher than 


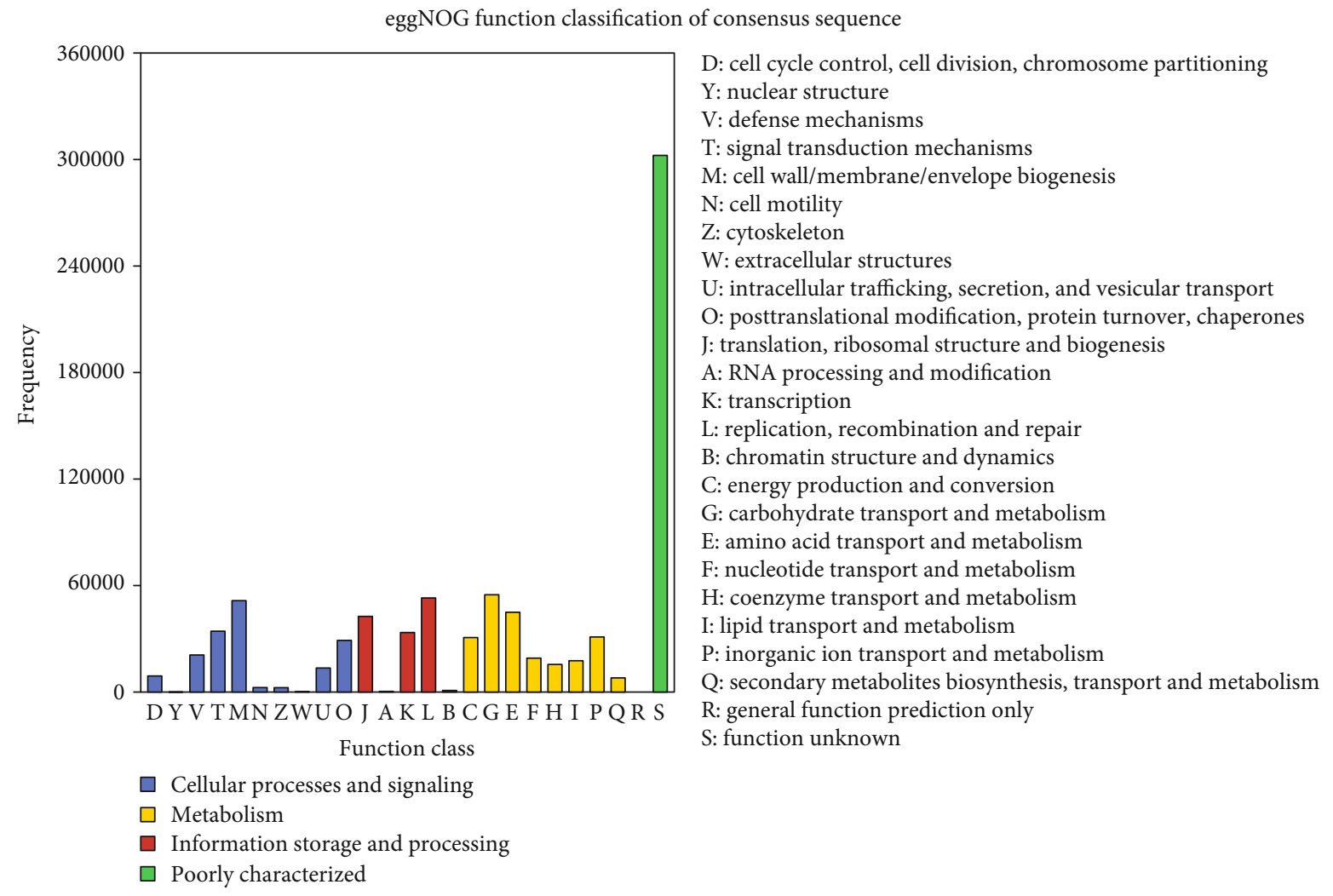

Figure 4: eggNOG functional annotation of identified genes.

TABLE 4: Top 10 enriched KEGG pathways.

\begin{tabular}{lcc}
\hline Pathway & Description & Number of genes \\
\hline ko01230 & Biosynthesis of amino acids & 26,409 \\
ko00230 & Purine metabolism & 18,237 \\
ko01200 & Carbon metabolism & 17,820 \\
ko00240 & Pyrimidine metabolism & 16,406 \\
ko02010 & ABC transporters & 14,061 \\
ko00500 & Starch and sucrose metabolism & 10,978 \\
k000520 & Amino sugar and nucleotide sugar metabolism & 10,636 \\
ko02024 & Quorum sensing & 10,401 \\
ko00970 & Aminoacyl-tRNA biosynthesis & 10,392 \\
k003010 & Ribosome & 10,165 \\
\hline
\end{tabular}

that reported for yaks in the same environment [25]. The distribution of intestinal bacteria-especially the $F / B$ ratio-has been linked to obesity in humans, which is thought to be related to the ability of Firmicutes to more effectively absorb calories from food $[26,27]$. As an adaptation to the highaltitude environment, high intestinal Firmicutes and Fibrobacteres contents can help cattle-yaks digest crude fiber material and produce a large amount of short-chain fatty acids that are utilized by the body for energy. Many microbial species associated with lignin and cellulose degradation were present in the rumen of adult cattle-yaks including $R$. flavefaciens, $R$. albus, F. succinogenes, and P. ruminicola $[28,29]$, and their abundance was similar between the two age groups.
Some studies have reported that $F$. succinogenes is the most highly represented of these species $[17,30]$, while others have shown that $R$. albus is more abundant $[31,32]$. F. succinogenes functions in the degradation of plant cellulose [3335]; we found here that F. succinogenes was the predominant species in the rumen of adult cattle-yaks whereas $R$. albus was the least abundant, which may be related to the grazing state of cattle-yaks in the plateau and long-term natural foraging behavior.

In our functional analysis of 1,281,665 nonredundant genes, 145,489 were annotated to the CAZy database. To date, 42 unique strains of cellulase- and GH-producing bacteria have been identified [36]. GHs hydrolyze glycosidic 
linkages between sugar molecules [3]. Most of the GHs identified in our study were oligosaccharide-degrading enzymes such as cellulase, hemicellulose, and pectinase that degrade the plant cell wall to produce oligosaccharides [37]. A higher proportion of oligosaccharide-degrading enzymes are associated with an increased rate of monosaccharide and VFA generation, which promotes nutrient absorption and utilization by ruminants [5]. In this study, the most highly represented $\mathrm{GH}$ family was $\mathrm{GH} 2$ (which comprises $\beta$-Dgalactosidase, $\beta$-glucuronidase, $\beta$-D-mannosidase, and exo- $\beta$-glucosaminidase [38]) followed by the GH43 (which includes $\beta$-xylosidase, $\beta$-1,3-xylosidase, $\alpha$-L-arabinofuranosidase, arabinanase, xylanase, galactan 1 , and 3 - $\beta$-galactosidase). Our results are in accordance with the finding that $\mathrm{GH} 3$ and $\mathrm{GH} 2$ are the predominant GHs in the rumen of Indian buffalo [3, 35, 36]. GTs catalyze the binding of sugars to glycosyl groups; we found that GT2, GT4, GT27, GT5, and GT41 families were the most highly represented in the rumen of cattle-yaks. Most of the CE and CBM genes belonged to the CE1, CE7, CE10, CBM6, CBM32, and CBM50 families and included xylan esterases of the CE1 and CE7 families, which act on branched xylan [39, 40]. We also identified 29 and 415 genes encoding cohesin and docker, respectively, which are components of the fibrosome, a multienzyme complex on the cell surface that degrades lignocellulose [41]. A total of 80 cohesin and 188 docker genes have been identified in the rumen of cows [42].

The functional annotation based on the eggNOG database revealed that the nonredundant genes in the two different age groups were mainly related to carbohydrate transport and metabolism, which could reflect the grazing environment of cattle-yaks in the plateau that requires long-term feeding in natural pastures and resistance to roughage and stress. We also found that the number of genes involved in energy production and transformation, amino acid transport and metabolism, and inorganic ion transport and metabolism was higher in 3-year-old as compared to 2-year-old cattle-yaks. Thus, the adaptability of cattle-yaks to the plateau environment increases with age. Additionally, 234,104 genes were enriched in 369 KEGG pathways, with those related to small molecule metabolism such as amino acid biosynthesis and purine, carbon, and pyrimidine metabolism being the most highly represented.

\section{Conclusion}

The results of this study demonstrate that the rumen microbiome of cattle-yaks is essentially unchanged in adulthood. Firmicutes, Fibrobacteres, Euryarchaeota, Bacteroidetes, and Proteobacteria were the predominant bacterial groups, with Firmicutes showing the highest abundance. F. succinogenes was the most highly represented species. An analysis of gene function found that the rumen of adult cattle-yaks is enriched in enzymes associated with lignocellulose and cellulose degradation. These results provide new insight into the heterosis of cattle-yaks and physiologic adaptations in plateau animals.

\section{Data Availability}

All relevant data involved in this study are presented in the "Result" and supplementary materials.

\section{Conflicts of Interest}

The authors declare that the research was conducted in the absence of any commercial or financial relationships that could be construed as a potential conflict of interest.

\section{Authors' Contributions}

Yuzhu Sha, Xiu Liu, Yuzhu Luo, and Jiang Hu designed the study. Yuzhu Sha, Bingang Shi, Jiqing Wang, Shaobin Li, Renqing Dingkao, and Wei Zhang performed the experiments and collected samples. Yuzhu Sha and Xiu Liu analyzed the data and wrote the manuscript. All authors contributed to manuscript revision and read and approved the final version.

\section{Acknowledgments}

We thank the financial support from the Science and Technology Innovation Funds of Gansu Agricultural University, Special Funds for Discipline Construction (GSAU-XKJS2018-027).

\section{Supplementary Materials}

Additional file: species composition. It is divided into bacteria kingdom and archaea kingdom and the composition and content of microorganisms in phylum-class-order-family-genus-species. CAZy database. GH family, GT family, CE family, and CBM family member composition and gene number. Figure S1: functional abundance of CAZy at different ages. Figure S2: functional abundance of eggNOG in different age groups. Figure S3: KEGG functional abundance in different age groups. Figure S4: KEGG pathway annotation. (Supplementary Materials)

\section{References}

[1] J. M. Brulc, D. A. Antonopoulos, M. E. Berg Miller et al., "Gene-centric metagenomics of the fiber-adherent bovine rumen microbiome reveals forage specific glycoside hydrolases," Proceedings of the National Academy of Sciences, vol. 106, no. 6, pp. 1948-1953, 2009.

[2] C. W. Forsberg, K. J. Cheng, and B. A. White, "Polysaccharide degradation in the rumen and large intestine," in Gastrointestinal Microbiology, R. I. Mackie and B. A. White, Eds., pp. 319379, Springer, Boston, MA, 1997.

[3] K. M. Singh, B. Reddy, D. Patel et al., "High potential source for biomass degradation enzyme discovery and environmental aspects revealed through metagenomics of Indian buffalo rumen," BioMed Research International, vol. 2014, Article ID 267189, 10 pages, 2014.

[4] P. R. Pandya, K. M. Singh, S. Parnerkar et al., "Bacterial diversity in the rumen of Indian Surti buffalo (Bubalus bubalis), assessed by $16 \mathrm{~S}$ rDNA analysis," Journal of Applied Genetics, vol. 51, no. 3, pp. 395-402, 2010. 
[5] E. Jami, A. Israel, A. Kotser, and I. Mizrahi, "Exploring the bovine rumen bacterial community from birth to adulthood," ISME Journal, vol. 7, no. 6, pp. 1069-1079, 2013.

[6] G. Wiener, H. Jianlin, and L. Ruijun, "Origins, domestication and distribution of yak," in The Yak, Second Edition, G. H. Wiener, H. Jianlin, and L. Ruijun, Eds., pp. 1-16, RAP Publication, Food and Agricultural Organization of the United Nations, Regional Office for Asia and the Pacific, Bangkok, Thailand, 2003.

[7] Z. Zhang, D. Xu, L. Wang et al., "Convergent evolution of rumen microbiomes in high-altitude mammals," Current Biology, vol. 26, no. 14, pp. 1873-1879, 2016.

[8] H. Li and R. Durbin, "Fast and accurate short read alignment with Burrows-Wheeler transform," Bioinformatics, vol. 25, no. 14, pp. 1754-1760, 2009.

[9] W. Zhu, A. Lomsadze, and M. Borodovsky, "Ab initio gene identification in metagenomic sequences," Nucleic Acids Research, vol. 38, no. 12, 2010.

[10] G. Flachowsky, "Rumen Microbiology: Burk A Dehority (Ed.), Nottingham University Press, Nottingham, NG11 OAX, UK, 2003, Hardcover, ISBN 1-897676-99-9, £ 40, 372 pp."” Animal Feed Science and Technology, vol. 113, no. 1-4, pp. 253-254, 2004.

[11] D. Xue, H. Chen, F. Chen et al., "Analysis of the rumen bacteria and methanogenic archaea of yak (Bos grunniens) steers grazing on the Qinghai-Tibetan Plateau," Livestock Science, vol. 188, pp. 61-71, 2016.

[12] N. Abdul Rahman, D. H. Parks, I. Vanwonterghem, M. Morrison, G. W. Tyson, and P. Hugenholtz, "A phylogenomic analysis of the bacterial phylum Fibrobacteres," Frontiers in Microbiology, vol. 6, 2016.

[13] P. B. Eckburg, E. M. Bik, C. N. Bernstein et al., "Diversity of the human intestinal microbial flora," Science, vol. 308, no. 5728, pp. 1635-1638, 2005.

[14] R. E. Ley, P. J. Turnbaugh, S. Klein, and J. I. Gordon, "Human gut microbes associated with obesity," Nature, vol. 444, no. 7122, pp. 1022-1023, 2006.

[15] J. Qin, R. Li, J. Raes et al., "A human gut microbial gene catalogue established by metagenomic sequencing," Nature, vol. 464, no. 7285, pp. 59-65, 2010.

[16] H. M. Tun, M. S. Brar, N. Khin et al., "Gene-centric metagenomics analysis of feline intestinal microbiome using 454 junior pyrosequencing," Journal of Microbiological Methods, vol. 88, no. 3, pp. 369-376, 2012.

[17] K. Tajima, S. Arai, K. Ogata et al., "Rumen bacterial community transition during adaptation to high-grain diet," Anaerobe, vol. 6, no. 5, pp. 273-284, 2000.

[18] K. M. Singh, V. B. Ahir, A. K. Tripathi et al., "Metagenomic analysis of Surti buffalo (Bubalus bubalis) rumen: a preliminary study," Molecular Biology Reports, vol. 39, no. 4, pp. 4841-4848, 2012.

[19] F. Armougom and D. Raoult, "Use of pyrosequencing and DNA barcodes to monitor variations in Firmicutes and Bacteroidetes communities in the gut microbiota of obese humans," BMC Genomics, vol. 9, no. 1, p. 576, 2008.

[20] D. Mariat, O. Firmesse, F. Levenez et al., "The Firmicutes/Bacteroidetes ratio of the human microbiota changes with age," BMC Microbiol, vol. 9, no. 1, p. 123, 2009.

[21] W. Guo, Y. Li, L. Wang et al., "Evaluation of composition and individual variability of rumen microbiota in yaks by $16 \mathrm{~S}$
rRNA high-throughput sequencing technology," Anaerobe, vol. 34, pp. 74-79, 2015.

[22] H. Li, J. Qu, T. Li et al., "Diet simplification selects for high gut microbial diversity and strong fermenting ability in highaltitude pikas," Applied Microbiology and Biotechnology, vol. 102, no. 15, pp. 6739-6751, 2018.

[23] A. R. Moschen, V. Wieser, and H. Tilg, "Dietary factors: major regulators of the gut's microbiota," Gut and Liver, vol. 6, no. 4, pp. 411-416, 2012.

[24] S. C. Fernando, H. T. Purvis, F. Z. Najar et al., "Rumen microbial population dynamics during adaptation to a high-grain diet," Applied and Environmental Microbiology, vol. 76, no. 22, pp. 7482-7490, 2010.

[25] J. Xin, Z. Chai, C. Zhang et al., "Comparing the microbial community in four stomach of dairy cattle, yellow cattle and three yak herds in Qinghai-Tibetan Plateau," Frontiers in Microbiology, vol. 10, 2019.

[26] N. R. Parmar, J. V. Solanki, A. B. Patel et al., "Metagenome of Mehsani buffalo rumen microbiota: an assessment of variation in feed-dependent phylogenetic and functional classification," Journal of Molecular Microbiology and Biotechnology, vol. 24, no. 4, pp. 249-261, 2014.

[27] R. E. Ley, P. J. Turnbaugh, S. Klein, and J. I. Gordon, "Microbial ecology: human gut microbes associated with obesity," Nature, vol. 444, no. 7122, pp. 1022-1023, 2006.

[28] H. J. Flint and E. A. Bayer, "Plant cell wall breakdown by anaerobic microorganisms from the mammalian digestive tract," Annals of the New York Academy of Sciences, vol. 1125, no. 1, pp. 280-288, 2008.

[29] H. J. Flint, E. A. Bayer, M. T. Rincon, R. Lamed, and B. A. White, "Polysaccharide utilization by gut bacteria: potential for new insights from genomic analysis," Nature Reviews Microbiology, vol. 6, no. 2, pp. 121-131, 2008.

[30] C. Martin, L. Millet, G. Fonty, and B. Michalet-Doreau, "Cereal supplementation modified the fibrolytic activity, but not the structure of the cellulolytic bacterial community associated with, rumen solid digesta," Reproduction Nutrition Development, vol. 41, no. 5, pp. 413-424, 2001.

[31] P. J. Weimer, G. C. Waghorn, C. L. Odt, and D. R. Mertens, "Effect of diet on populations of three species of ruminal cellulolytic bacteria in lactating dairy cows," Journal of Dairy Science, vol. 82, no. 1, pp. 122-134, 1999.

[32] D. O. Krause and J. B. Russell, "An rRNA approach for assessing the role of obligate amino acid-fermenting bacteria in ruminal amino acid deamination," Applied and Environmental Microbiology, vol. 62, no. 3, pp. 815-821, 1996.

[33] E. Ransom-Jones, D. L. Jones, A. Edwards, and J. E. McDonald, "Distribution and diversity of members of the bacterial phylum _Fibrobacteres_ in environments where cellulose degradation occurs," Systematic and Applied Microbiology, vol. 37, no. 7, pp. 502-509, 2014.

[34] E. Ransom-Jones, D. L. Jones, A. J. McCarthy, and J. E. McDonald, "The Fibrobacteres: an important phylum of cellulosedegrading bacteria," Microbial Ecology, vol. 63, no. 2, pp. 267-281, 2012.

[35] E. Rosenberg, "The phylum Fibrobacteres," in The Prokaryotes, pp. 641-642, Springer, Berlin, 2014.

[36] Z. Bashir, V. K. Kondapalli, N. Adlakha et al., "Diversity and functional significance of cellulolytic microbes living in termite, pill-bug and stem-borer guts," Scientific Reports, vol. 3, no. 1,2013 . 
[37] R. D. Stewart, M. D. Auffret, A. Warr et al., "Assembly of 913 microbial genomes from metagenomic sequencing of the cow rumen," Nature Communications, vol. 9, no. 1, 2018.

[38] A. S. Nascimento, J. R. Muniz, R. Aparício, A. M. Golubev, and I. Polikarpov, "Insights into the structure and function of fungal $\beta$-mannosidases from glycoside hydrolase family 2 based on multiple crystal structures of the Trichoderma harzianum enzyme," FEBS Journal, vol. 281, no. 18, pp. 4165-4178, 2014.

[39] D. D. Patel, A. K. Patel, N. R. Parmar et al., "Microbial and carbohydrate active enzyme profile of buffalo rumen metagenome and their alteration in response to variation in the diet," Gene, vol. 545, no. 1, pp. 88-94, 2014.

[40] H. Zhang, J. Li, J. Wang, Y. Yang, and M. Wu, "Determinants for the improved thermostability of a mesophilic family 11 xylanase predicted by computational methods," Biotechnology for Biofuels, vol. 7, no. 1, p. 3, 2014.

[41] J. E. Hyeon, S. D. Jeon, and S. O. Han, "Cellulosome-based, Clostridium-derived multi-functional enzyme complexes for advanced biotechnology tool development: advances and applications," Biotechnology Advances, vol. 31, no. 6, pp. 936-944, 2013.

[42] M. Hess, A. Sczyrba, R. Egan et al., "Metagenomic discovery of biomass-degrading genes and genomes from cow rumen," Science, vol. 331, no. 6016, pp. 463-467, 2011. 